\title{
Exploring the Dynamical Behavior of Information Diffusion in D2D Communication Environment
}

\author{
Zufan Zhang ${ }^{(D,}{ }^{1,2,3}$ Anqi Liu, ${ }^{1}$ Yinxue Yi, ${ }^{1}$ and Maobin Yang ${ }^{1}$ \\ ${ }^{1}$ School of Communication and Information Engineering, Chongqing University of Posts and Telecommunications, \\ Chongqing 400065, China \\ ${ }^{2}$ Chongqing Key Laboratory of Mobile Communications Technology, Chongqing 400065, China \\ ${ }^{3}$ Engineering Research Center of Mobile Communications, Ministry of Education, Chongqing 400065, China \\ Correspondence should be addressed to Zufan Zhang; zhangzf@cqupt.edu.cn
}

Received 3 August 2020; Revised 31 August 2020; Accepted 10 September 2020; Published 22 September 2020

Academic Editor: Chunming Zhang

Copyright (c) 2020 Zufan Zhang et al. This is an open access article distributed under the Creative Commons Attribution License, which permits unrestricted use, distribution, and reproduction in any medium, provided the original work is properly cited.

\begin{abstract}
This paper is dedicated to exploring the dynamical behavior of information diffusion in the Device-to-Device (D2D) communication environment for information security, so as to study how to accelerate the dissemination of beneficial information and curb the spread of malicious information. A mathematical model of information diffusion considering the combined impact of user awareness and social tie between users is proposed. The equilibrium of the model and its stability are fully analyzed. Very importantly, there is a unique (viral) equilibrium that is globally asymptotically stable without any preconditions. This means that the spread of malicious information in the D2D communication environment cannot be completely eliminated whatever measures are taken, but its diffusion scale can be controlled by adjusting the value of the equilibrium, and then the goal of pursuing the best control effect at the minimum cost can be achieved. In the same way, the dissemination scale of beneficial information can be expanded. Finally, the obtained main theoretical results are illustrated by some examples, and some suggestions are also given.
\end{abstract}

\section{Introduction}

The Device-to-Device (D2D) communication is a promising approach to address the growing demand for data transmission, which provides a high transmission rate, low latency, and low power consumption [1]. In recent years, combining social networks with D2D communications has become a hotspot in the field of D2D research $[2,3]$. With the explosive growth of information in social networks, higher requirements for efficiency and security of D2D communications are proposed [4]. Exploring information diffusion in the D2D communication environment will help solve these problems.

In the D2D communication environment, users interact frequently on social networks [5], thus derivating social attributes such as social tie and intimacy $[6,7]$. As the main descriptors of information, individuals and their social attributes are closely related to its diffusion. Some previous work has explored the influence of social attributes on information diffusion. Gan et al. [8] proposed a diffusion model affected by user behavior in D2D communications. The results point out that the social attributes of users and information can improve the dissemination effect. Meng et al. [9] utilized the social tie to select peers for improving the performance of D2D link cooperative communication. Zhang et al. [10] considered that the social tie decay with time and the priority schemes are selected by maximizing the strength of social tie for improving the information transmission rate.

The exploration of social attributes has effectively improved the efficiency of information dissemination in D2D communications. However, in the real life, all kinds of information including beneficial, malicious, and generic proliferate competitively on social networks [11-13]. For the information such as computer viruses [14, 15], malware $[16,17]$, and rumors $[18,19]$, it is important to consider taking necessary measures to suppress their spread. Meanwhile, the dissemination of alerts [20], safety publicity, 
and emergencies are also expected to accelerate [21]. In addition, the user awareness plays an important role in the dissemination of different information. For example, when a user realizes the existence of malicious information, it will inform others about the information dissemination, and the user who knows the message can take measures to refuse to receive it. The classification of information has also considered by scholars. Wu et al. [22] proposed a random diffusion model to explore the minimization of the diffusion of nonsensitive information while suppressing the spread of sensitive information. Yang et al. [23] proposed a linear threshold model to explore how to suppress rumors by spreading positive information. Yang et al. [24] proposed an individual competition diffusion model, which discussed the influence of two spreading networks on the competition and diffusion of two conflicting messages.

In addition to the abovementioned work, epidemic models are often used to study information diffusion $[25,26]$. Lin et al. [27] proposed a susceptible-wanderinginfected-recovery (SWIR) model to describe the influence of individual psychological state change on the dissemination of fraud information and studied the control problem of fraud information with personal loss as a constraint. Sun et al. [28] proposed an improved SIR model to analyze the competitive diffusion of positive and negative information and analyzed the stability of diffusion infection-free equilibrium. Yi et al. [29] introduced the uncertain individual called hesitator in the SIR model and utilized the selfconfirmation mechanism to describe the influence of social tie strength on user-forwarding behavior. Although these studies have modeled and analyzed the information diffusion, most of them mainly consider the diffusion behavior in social networks and assumed that users are the main senders and receivers. In the D2D communication environment, social and D2D physical networks jointly influence the dissemination of information, and the existing models cannot accurately describe this process.

Inspired by the above research studies, this paper explores the dynamical behavior of information diffusion in the D2D communication environment. Firstly, a mathematical model combined the effect of user awareness and social tie is proposed. Next, the global stability of equilibrium is analyzed, which shows that the spread of malicious information in the D2D communication environment cannot be completely eliminated, but the diffusion scale can be reduced to a certain level by adjusting the system parameters. Similarly, the beneficial information can also be expanded. Finally, the obtained main theoretical results are verified by some examples, and some directional suggestions are recommended to accelerate the dissemination of beneficial information or to curb the spread of malicious information.

The subsequent materials are organized as follows. Section 2 briefly describes the scenario of D2D communication environment. Sections 3 and 4 propose and analyze the mathematical model of information diffusion, respectively. Some discussions are made in Section 5. Finally, Section 6 summarizes this work.

\section{Scenario Description}

This paper mainly explores the dynamical behavior of information diffusion in the $\mathrm{D} 2 \mathrm{D}$ communication environment. Figure 1 briefly shows the information diffusion diagram. In a cellular network, there are traditional cellular users $C=\left(C_{1}, C_{2}\right)$ to communicate through the Base Station (BS). Since the devices $d_{1}$ and $d_{2}$ with $\mathrm{D} 2 \mathrm{D}$ communication function can directly establish D2D peer-to-peer links, the D2D users $D=\left(D_{1}, \ldots, D_{7}\right)$ can also directly establish peerto-peer sessions to send and receive the information. In particular, a single user can establish a peer-to-peer session with a single user, such as $D_{4} \longrightarrow D_{5}$, or can establish peerto-peer sessions with multiple users, such as $\left(D_{1}, D_{3}\right) \longrightarrow D_{2}$. Moreover, in some cases, a third-party relay user can perform multihop communication such as $D_{6} \longrightarrow R_{1} \longrightarrow D_{7}$, where $R_{1}$ acts as a relay. This is suitable for users who are relatively far away to communicate. Meanwhile, whether the D2D link can be successfully established is also related to the social tie between users, such as $p_{1}, p_{2}$, and $p_{3}$. Next, this paper will establish a mathematical model based on this scenario to study the dynamical behavior of information diffusion.

\section{Mathematical Formulation}

Based on the scenario description, for any given information, all users in the D2D communication environment are in one of the following two states:

Unknown (U): the user does not know the information.

Known $(\mathrm{K})$ : the user knows the information through the other users or the corresponding devices. Once the corresponding devices receive the information, the user is sure to know the information.

According to whether the devices receive the information or not, all devices in the D2D communication environment are also in one of the two states: Infected (I) or Susceptible (S). Here, let us regard the user and the corresponding devices as a node; then, the node is in one of the following three states:

US : the user does not know the information, and the corresponding devices have never received the information

KS : the user knows the information through the other users, but the corresponding devices do not receive the information

$\mathrm{KI}$ : the user knows the information, and the corresponding devices receive the information

From the scenario and node sate description, Figure 2 gives the state transfer diagram, and the corresponding details are as follows:

At any time, the establishing probabilities of a new D2D link between US, KS, and KI nodes are $\delta_{1}, \delta_{2}$, and $\delta_{3}$, respectively. 


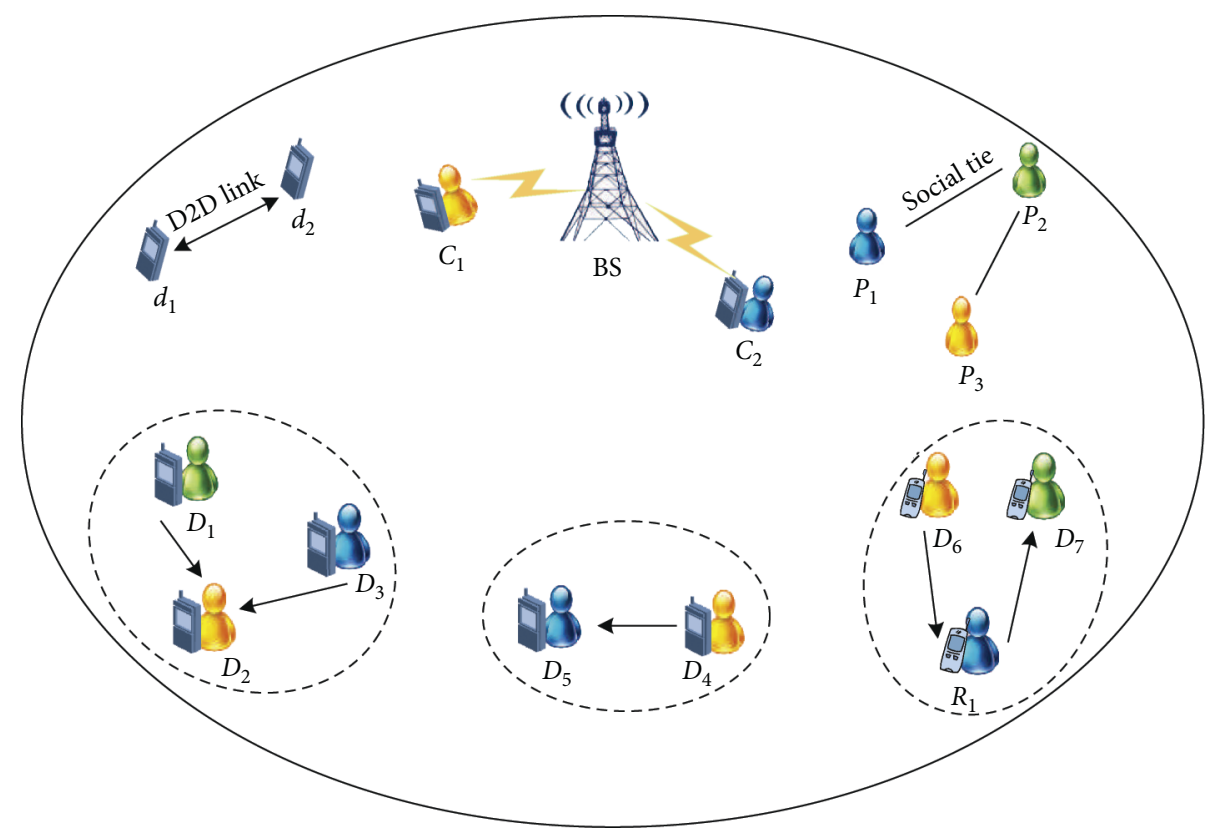

Figure 1: The diagram of information diffusion in the D2D communication environment.

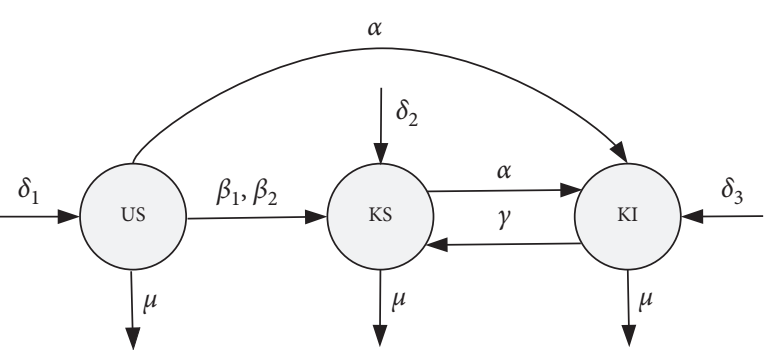

Figure 2: The state transfer diagram.

At any time, the break probability of D2D link between nodes is $\mu$.

Due to the effect of social tie between users, at any time, the probability that the US node obtains the message about information dissemination from the KS or KI node is $\beta_{1}$ or $\beta_{2}$.

At any time, a node in the US or KS state receives the information with probability $\alpha$.

Due to the impact of user awareness, at any time, a node in the KI state deletes the received information with probability $\gamma$.

Let $x(t), y(t)$, and $z(t)$ represent the number of US, KS, and KI nodes at time $t$, respectively, and let $N(t)$ stand for the total number of all nodes; then, $N(t)=x(t)$ $+y(t)+z(t)$. For brevity, $x(t), y(t), z(t)$, and $N(t)$ will be abbreviated as $x, y, z$, and $N$, respectively.

Collecting the above descriptions, the mathematical model of information diffusion of the state transfer diagram in Figure 2 can be expressed as follows:

$$
\left\{\begin{array}{l}
\frac{d x}{d t}=\delta_{1}-\beta_{1} x y-\beta_{2} x z-\alpha x z-\mu x, \\
\frac{d y}{d t}=\delta_{2}+\beta_{1} x y+\beta_{2} x z+\gamma z-\alpha y z-\mu y, \\
\frac{d z}{d t}=\delta_{3}+\alpha x z+\alpha y z-\gamma z-\mu z,
\end{array}\right.
$$

with the initial condition $(x(0), y(0), z(0)) \in R_{+}^{3}$.

Noting $N=x+y+z$, then adding the two sides of system (1) gives $d N / d t=\delta-\mu N$, and system (1) can be transformed into the following equivalent system:

$$
\left\{\begin{array}{l}
\frac{d N}{d t}=\delta-\mu N, \\
\frac{d y}{d t}=\delta_{2}+\beta_{1} y(N-y-z)+\beta_{2} z(N-y-z)+\gamma z-\alpha z y-\mu y, \\
\frac{d z}{d t}=\delta_{3}+\alpha z(N-z)-\gamma z-\mu z,
\end{array}\right.
$$

with $\delta=\delta_{1}+\delta_{2}+\delta_{3}$ and the initial condition $(N(0), y(0), z(0)) \in R_{+}^{3}$.

From the first equation of system (2), one can obtain

$$
N=N^{*}+C e^{-\mu t}
$$

where $N^{*}=\delta / \mu$ and $C \in R$.

From (3), one can get $\lim _{t \longrightarrow+\infty} N=N^{*}$. Then, system (2) can be simplified as the following limiting system [30]: 


$$
\left\{\begin{array}{l}
\frac{d y}{d t}=\delta_{2}+\beta_{1} y\left(N^{*}-y-z\right)+\beta_{2} z\left(N^{*}-y-z\right)+\gamma z-\alpha z y-\mu y, \\
\frac{d z}{d t}=\delta_{3}+\alpha z\left(N^{*}-z\right)-\gamma z-\mu z,
\end{array}\right.
$$

with the positively invariant region:

$$
\Omega=\left\{(y, z) \in R_{+}^{2}: y+z \leq N^{*}\right\} .
$$

In what follows, it suffices to study the dynamical behavior of system (4) with respect to $\Omega$.

\section{Model Analysis}

Before proceeding to the next analysis of the model, let us first introduce some basic terminology concepts, which will help explain the motivation and main research content of this paper.

Dynamical behavior: the behavior of dynamical systems. Here, this paper discusses the stability of equilibrium of system (4).

Equilibrium: it is a fixed point of a dynamical system. In this paper, it represents the possible final scale of information diffusion. The viral equilibrium means that the information diffusion component is not zero.

Stability: the final motion trend of the orbit of a dynamical system. In this paper, the local stability and global stability of equilibrium of system (4) are both analyzed.

4.1. Equilibrium. First of all, let us discuss the equilibrium of system (4).

Theorem 1. There is a unique (viral) equilibrium $E^{*}\left(y^{*}, z^{*}\right)$ in system (4), where

$$
\begin{aligned}
& z^{*}=\frac{w_{0}+\sqrt{w_{0}^{2}+4 \alpha \delta_{3}}}{2 \alpha}, \\
& y^{*}=\frac{-w_{1}+\sqrt{w_{1}^{2}+4 \beta_{1} w_{2}}}{2 \beta_{1}}, \\
& w_{0}=\alpha N^{*}-\gamma-\mu, \\
& w_{1}=\left(\beta_{2}+\alpha\right) z^{*}+\mu-\beta_{1}\left(N^{*}-z^{*}\right), \\
& w_{2}=\delta_{2}+\beta_{2} z^{*}\left(N^{*}-z^{*}\right)+\gamma z^{*} .
\end{aligned}
$$

Proof. Suppose that $(\widetilde{y}, \widetilde{z})$ is an equilibrium of system (4). Then,

$$
\left\{\begin{array}{l}
\delta_{2}+\beta_{1} \tilde{y}\left(N^{*}-\tilde{y}-\widetilde{z}\right)+\beta_{2} \tilde{z}\left(N^{*}-\tilde{y}-\tilde{z}\right)+\gamma \widetilde{z}-\alpha \widetilde{z} \tilde{y}-\mu \widetilde{y}=0 \\
\delta_{3}+\alpha \widetilde{z}\left(N^{*}-\widetilde{z}\right)-\gamma \widetilde{z}-\mu \widetilde{z}=0 .
\end{array}\right.
$$
btain

Solving the second equation of system (7), one can

$$
\widetilde{z}=\frac{\alpha N^{*}-\gamma-\mu+\sqrt{\left(\alpha N^{*}-\gamma-\mu\right)^{2}+4 \alpha \delta_{3}}}{2 \alpha}
$$

$$
\begin{aligned}
& =\frac{w_{0}+\sqrt{w_{0}^{2}+4 \alpha \delta_{3}}}{2 \alpha} \\
& =z^{*} .
\end{aligned}
$$

Next, putting (8) into the first equation of system (7), it can calculate that $\tilde{y}=y^{*}$. Therefore, the proof is complete.

4.2. Local Stability. Here, let us consider the local stability of $E^{*}$ of system (4).

Theorem 2. The unique (viral) equilibrium $E^{*}$ of system (4) is locally asymptotically stable.

Proof. The Jacobian matrix of system (4) at $E^{*}$ is

$$
J_{E^{*}}=\left(\begin{array}{cc}
w_{3} & w_{4} \\
0 & w_{5}
\end{array}\right)
$$

where

$$
\begin{aligned}
& w_{3}=\beta_{1}\left(N^{*}-y^{*}-z^{*}\right)-\beta_{1} y^{*}-\beta_{2} z^{*}-\alpha z^{*}-\mu, \\
& w_{4}=\beta_{2}\left(N^{*}-y^{*}-z^{*}\right)-\beta_{2} z^{*}-\left(\beta_{1}+\alpha\right) y^{*}+\gamma \\
& w_{5}=\alpha\left(N^{*}-z^{*}\right)-\alpha z^{*}-\gamma-\mu .
\end{aligned}
$$

Furthermore, the corresponding characteristic equation is

$$
\lambda^{2}+k_{1} \lambda+k_{2}=0
$$

where

$$
\begin{aligned}
& k_{1}=-\left(w_{3}+w_{5}\right), \\
& k_{2}=w_{3} w_{5} .
\end{aligned}
$$

From system (7), one can obtain

$$
\begin{aligned}
\alpha\left(N^{*}-z^{*}\right)= & \alpha\left(\frac{\gamma+\mu}{\alpha}-\frac{\delta_{3}}{\alpha z^{*}}\right)=\gamma+\mu-\frac{\delta_{3}}{z^{*}}, \\
\alpha z^{*}+\mu= & \beta_{1}\left(N^{*}-y^{*}-z^{*}\right) \\
& +\frac{1}{y^{*}}\left(\beta_{2} z^{*}\left(N^{*}-y^{*}-z^{*}\right)+\gamma z^{*}+\delta_{2}\right) .
\end{aligned}
$$

Thus, 


$$
\begin{aligned}
k_{1}= & \frac{1}{y^{*}}\left(\beta_{2} z^{*}\left(N^{*}-y^{*}-z^{*}\right)+\gamma z^{*}+\delta_{2}\right)+\beta_{1} y^{*}+\beta_{2} z^{*} \\
& +\alpha z^{*}+\frac{\delta_{3}}{z^{*}}>0, \\
k_{2}= & \left(\frac{1}{y^{*}}\left(\beta_{2} z^{*}\left(N^{*}-y^{*}-z^{*}\right)+\gamma z^{*}+\delta_{2}\right)+\beta_{1} y^{*}+\beta_{2} z^{*}\right) \\
& \cdot\left(\alpha z^{*}+\frac{\delta_{3}}{z^{*}}\right)>0 .
\end{aligned}
$$

As $k_{1}>0, k_{2}>0$, then it follows from the Hurwitz criterion that the real parts of both roots of the characteristic (11) are negative. Therefore, the claimed result follows from Lyapunov stability theorem [31].

4.3. Global Stability. Now, let us analyze the global stability of $E^{*}$ of system (4). The following two lemmas are helpful for addressing it.

Lemma 1. System (4) has no periodic orbital in $\Omega$.

Proof. Define

$$
\begin{aligned}
& G(y, z)= \delta_{2}+\beta_{1} y\left(N^{*}-y-z\right) \\
&+\beta_{2} z\left(N^{*}-y-z\right)+\gamma z-\alpha z y-\mu y, \\
& H(y, z)= \delta_{3}+\alpha z\left(N^{*}-z\right)-\gamma z-\mu z, \\
& B(y, z)=\frac{1}{y z} .
\end{aligned}
$$

Then,

$$
\frac{\partial(B G)}{\partial y}+\frac{\partial(B H)}{\partial z}=-\frac{\delta_{2}}{z y^{2}}-\frac{\delta_{3}}{y z^{2}}-\frac{\beta_{2}\left(N^{*}-z\right)+\gamma}{y^{2}}-\frac{\alpha}{y}-\frac{\beta_{1}}{z}<0 .
$$

Hence, the claimed result follows from the Bendixson-Dulac criterion [31].

Lemma 2. System (4) has no periodic orbit on the boundary of $\Omega$.

Proof. Assume that $(\tilde{y}, \widetilde{z})$ is an arbitrary point on the boundary of $\Omega$. Then, let us consider the following three cases.

Case 1: when $\widetilde{z}=0,0 \leq \tilde{y} \leq N^{*}$, then

$$
\left.\frac{d z}{d t}\right|_{(\tilde{y}, \tilde{z})}=\delta_{3}>0
$$

Case 2: when $\tilde{y}=0,0<\widetilde{z}<N^{*}$, then

$$
\left.\frac{d y}{d t}\right|_{(\tilde{y}, \tilde{z})}=\delta_{2}+\beta_{2} \widetilde{z}\left(N^{*}-\widetilde{z}\right)+\gamma \widetilde{z}>0
$$

Case 3: when $\widetilde{y}+\widetilde{z}=N^{*}, \widetilde{y} \neq 0, \widetilde{z} \neq 0$, then

$$
\begin{aligned}
\left.\frac{d(y+z)}{d t}\right|_{(\tilde{y}, \tilde{z})} & =\delta_{2}+\delta_{3}-\mu \widetilde{y}-\mu \widetilde{z} \\
& =\delta_{2}+\delta_{3}-\mu N^{*} \\
& =-\delta_{1}<0
\end{aligned}
$$

Thus, the above analysis indicates that there are no periodic orbits that pass through $(\widetilde{y}, \widetilde{z})$ for system (4) on the boundary of $\Omega$. The proof is complete.

According to Theorem 1, Lemmas 1 and 2, and combined with the generalized Poincare-Bendixson theorem [31], it can demonstrate the global stability of $E^{*}$ of system (4). The main result is as follows.

Theorem 3. $E^{*}$ is globally asymptotically stable with respect to $\Omega$.

\section{Discussions}

To verify the obtained main theoretical, the following examples are given.

Example 1. Illustrate the effect of different initial conditions: $(x(0), y(0), z(0))=(450,40,10) ;(x(0), y(0), z(0))=$ $(400,95,5) ; \quad(x(0), y(0), z(0))=(300,190,10) \quad$ on system (1) with the common system parameters: $\mu=0.004, \alpha=$ $0.0002, \beta_{1}=0.0002, \beta_{2}=0.00015, \delta_{1}=0.6, \quad \delta_{2}=0.5, \delta_{3}=$ 0.4 , and $\gamma=0.03$.

Figure 3 shows the change trend of the number about each node of system (1) under different initial conditions. It can be seen that, although the initial conditions are different, the number of nodes in each state eventually tends to the same level. This is consistent with Theorem 3. The final information diffusion scale in the D2D communication environment is not affected by the initial condition. Besides, it can be found that the amount of US nodes rapidly drops to a low level and does not increase again. This is because the known users will no longer become the unknown state under the influence of user awareness, which is in line with reality.

Example 2. Illustrate the impact of different system parameters $\gamma$ on the number of $\mathrm{KS}$ and KI nodes with $\alpha=0.0002, \beta_{1}=0.0002, \beta_{2}=0.00015, \mu=0.004, \delta_{1}=0.6, \delta_{2}$ $=0.5$, and $\delta_{3}=0.4$ and the initial condition $(x(0), y$ $(0), z(0))=(400,95,5)$.

Figure 4 illustrates the effect of different $\gamma$ on the number of KS and KI nodes. It can be seen that the increase of $\gamma$ will reduce the number of infected nodes and can reach a lower level. This is because $\gamma$ represents the probability of the user deleting the information. Although the information is 


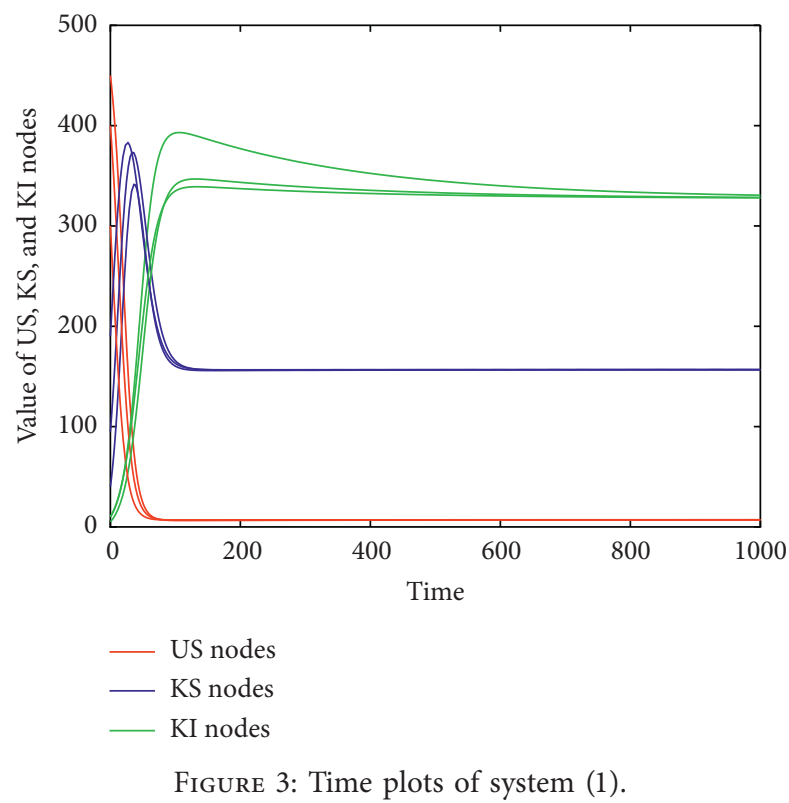

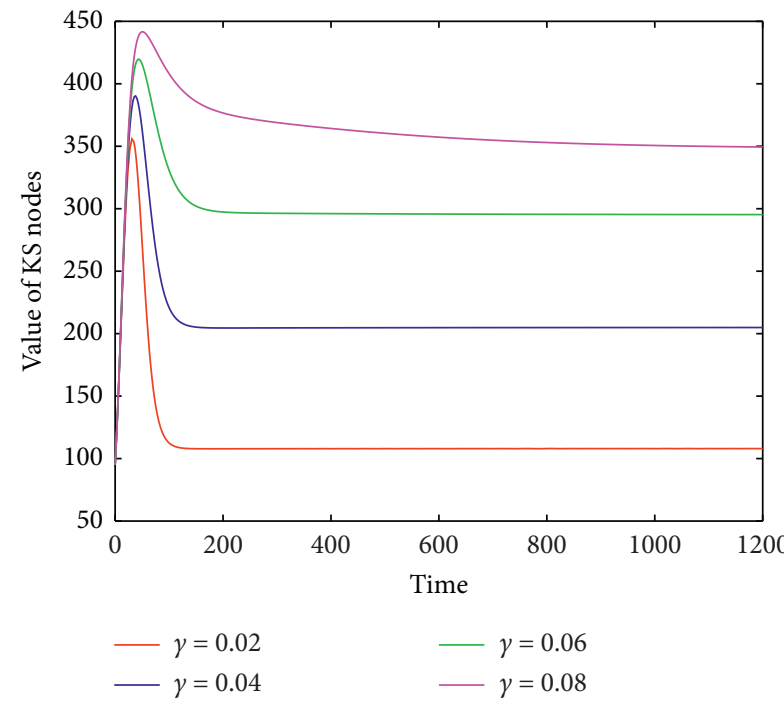

(a)

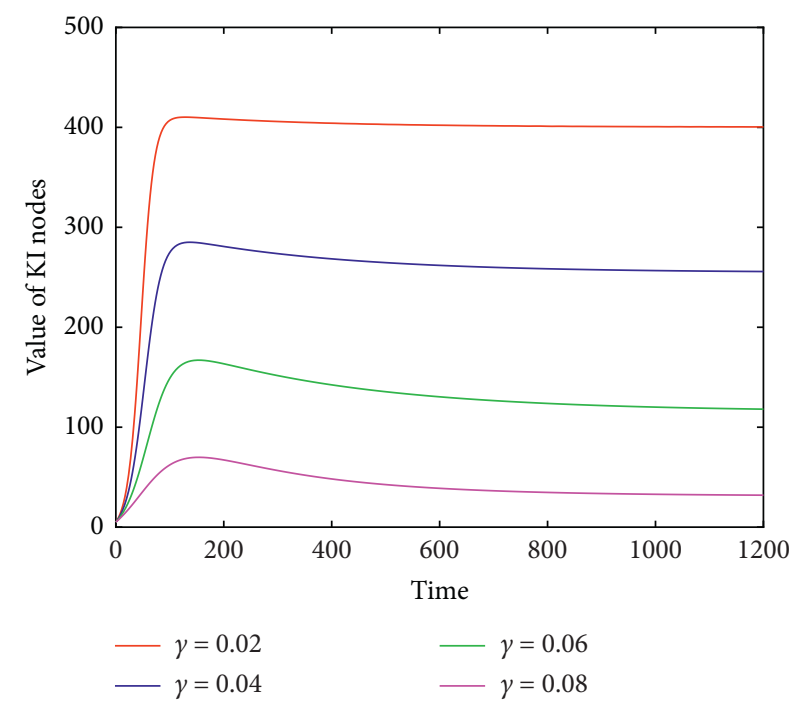

(b)

FIgURE 4: The impact of $\gamma$ on the number of KS and KI nodes.

deleted from the device, the user knows the information, which means that the information has been diffused. For malicious information, the rapid deletion of information can avoid secondary diffusion, so the security awareness of users and the necessary protection of the device are particularly important.

In addition, Theorem 3 reveals that the information will be persist in the $\mathrm{D} 2 \mathrm{D}$ communication environment, and if the information is malicious, it cannot be completely eliminated whatever measures are taken. So, it is essential to study how to control the spread of malicious information. From Theorem 1, one can observe that the value of the equilibrium can be adjusted by changing the system parameters, and then the following result is obtained.

Theorem 4. Assume that $U \in N_{+}$. Then, $z^{*} \leq U$ if and only if

$$
U^{2}-w_{0} U-4 \alpha \delta_{3} \geq 0
$$

where $w_{0}$ has been given in (6).

Proof. From (6), one can obtain

$$
z^{*}=\frac{w_{0}+\sqrt{w_{0}^{2}+4 \alpha \delta_{3}}}{2 \alpha} .
$$


Then,

$$
\frac{w_{0}+\sqrt{w_{0}^{2}+4 \alpha \delta_{3}}}{2 \alpha} \leq U .
$$

By a calculation, the claimed result follows.

Theorem 4 shows that the diffusion scale of malicious information can be controlled by adjusting the value of the equilibrium, and then the goal of pursuing the best control effect at the minimum cost can be achieved. In the same way, the dissemination scale of beneficial information can be expanded.

Example 3. Illustrate the information diffusion scale that can be achieved by adjusting system parameters. Taking the number of KI nodes as an indicator, in the case of ensuring (20) and $U<500$, the following two sets of conditions are made.

$$
\begin{aligned}
& \text { Condition 1: } \gamma=0.01, \alpha=0.0002, \beta_{1}=0.0002, \beta_{2}= \\
& 0.00015, \mu=0.004, \delta_{1}=0.6, \delta_{2}=0.5 \text {, and } \delta_{3}=0.4 \\
& \text { Condition 2: } \gamma=0.07, \alpha=0.0001, \beta_{1}=0.0002, \beta_{2}= \\
& 0.00015, \mu=0.005, \delta_{1}=0.6, \delta_{2}=0.5 \text {, and } \delta_{3}=0.4
\end{aligned}
$$

Figure 5 displays that, by adjusting the system parameters, the proportion of KI nodes can reach more than $90 \%$, and in the same way the proportion of KI nodes can reach an extremely low level. This also implies that the diffusion range of information can reach more than $90 \%$, and it can also be suppressed to a level that tends to disappear by means of the corresponding method. Furthermore, the related suggestions are as follows:

Use the core network in the D2D communication environment as a relay to broadcast the beneficial information

Increase mutual authentication of users in the D2D communication environment to improve connection reliability

Enhance the user safety awareness and install protection software in $\mathrm{D} 2 \mathrm{D}$ devices

Allow user permissions in the D2D communication environment to be revoked, and timely revoke user permissions or break the D2D link when the user is malicious

\section{Conclusions}

In this paper, a mathematical model of information diffusion in the D2D communication environment with the combined impact of user awareness and social tie between users has been developed for information security. The equilibrium of the model and its stability have been fully analyzed. Very importantly, there is a unique (viral) equilibrium that is globally asymptotically stable without any preconditions. Through the theoretical analysis, adjusting the system parameters can accelerate the dissemination of beneficial information or curb the spread of malicious information. Finally, some examples and suggestions have been given.

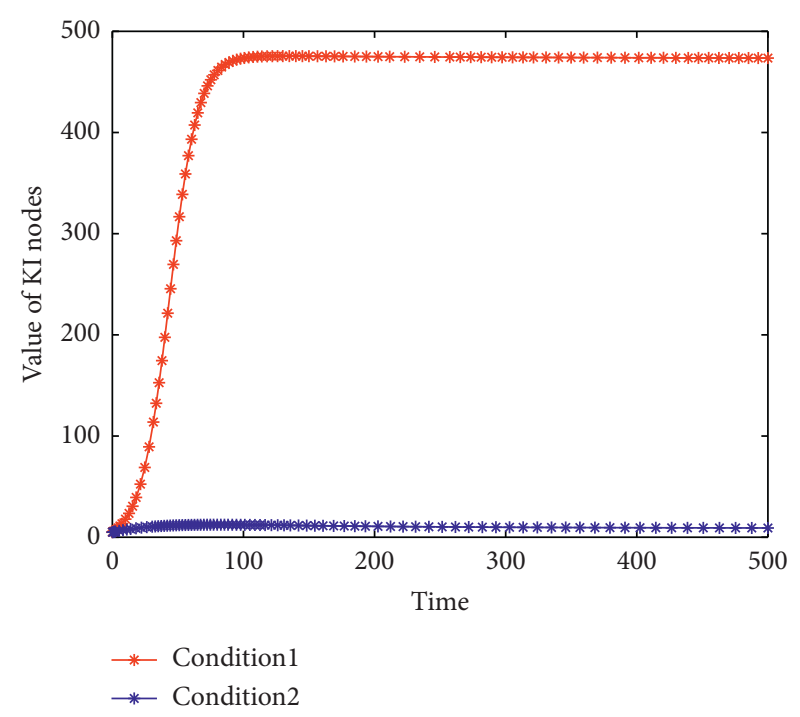

Figure 5: Time plots of KI nodes.

Although this paper does theoretical analysis of D2D communications and gives some practical measures, the established model is still relatively simple and lacks the support of actual data. In the next step, we can build a more complex information dissemination model, and it is best to separate people and equipment to consider the mutual influence of each other. In addition, since it involves real data issues, we will consider applying big data, deep learning, blockchain, and other technologies [32-34] to information dissemination research.

\section{Data Availability}

Data sharing is not applicable to this article as no datasets were generated.

\section{Conflicts of Interest}

All authors declare no conflicts of interest.

\section{Authors' Contributions}

The authors claim that the research was realized in collaboration with the same responsibility. All authors read and approved the last version of the manuscript.

\section{Acknowledgments}

This work was supported by Natural Science Foundation of China (Grant nos. 61702066 and 11747125), Major Project of Science and Technology Research Program of Chongqing Education Commission of China (Grant no. KJZDM201900601), Chongqing Research Program of Basic Research and Frontier Technology (Grant nos. cstc2017jcyjAX0256 and cstc2018jcyjAX0154), and Project Supported by Chongqing Municipal Key Laboratory of Institutions of Higher Education (Grant no. cqupt-mct-201901). 


\section{References}

[1] R. I. Ansari, C. Chrysostomou, S. A. Hassan et al., "5G D2D networks: techniques, challenges, and future prospects," IEEE Systems Journal, vol. 12, no. 4, pp. 3970-3984, 2018.

[2] M. Nitti, G. Stelea, V. Popescu, and M. Fadda, "When social networks meet D2D communications: a survey," Sensors, vol. 19, no. 2, pp. 396-412, 2019.

[3] Y. He, F. R. Yu, N. Zhao, and H. Yin, "Secure social networks in $5 \mathrm{G}$ systems with mobile edge computing, caching, and device-to-device communications," IEEE Wireless Communications, vol. 25, no. 3, pp. 103-109, 2018.

[4] F. H. Kumbhar, N. Saxena, and A. Roy, "Reliable relay: autonomous social D2D paradigm for 5G LoS communications," IEEE Communications Letters, vol. 21, no. 7, pp. 1593-1596, 2017.

[5] Y. Yi, Z. Zhang, L. T. Yang, C. Gan, X. Deng, and L. Yi, "Reemergence modeling of intelligent information diffusion in heterogeneous social networks: the dynamics perspective," IEEE Transactions on Network Science and Engineering, vol. 1, 2020.

[6] H. Xia, L. Li, X. Cheng, C. Liu, and T. Qiu, "A dynamic virus propagation model based on social attributes in city IoTs," IEEE Internet of Things Journal, vol. 1, 2020.

[7] M. Cai, W. Wang, Y. Cui, and H. E. Stanley, "Multiplex network analysis of employee performance and employee social relationships," Physica A: Statistical Mechanics and Its Applications, vol. 490, pp. 1-12, 2018.

[8] C. Gan, X. Li, L. Wang, and Z. Zhang, "The impact of user behavior on information diffusion in D2D communications: a discrete dynamical model," Discrete Dynamics in Nature and Society, vol. 2018, p. 9, Article ID 3745769, 2018.

[9] Y. Meng, C. Jiang, H.-H. Chen, and Y. Ren, "Cooperative device-to-device communications: social networking perspectives," IEEE Network, vol. 31, no. 3, pp. 38-44, 2017.

[10] Z. Zhang and L. Wang, "Social tie-driven content priority scheme for D2D communications," Information Sciences, vol. 480, pp. 160-173, 2019.

[11] Q. Li, Z. Wang, B. Wu, and Y. Xiao, "Competition and cooperation: dynamical interplay diffusion between social topic multiple messages in multiplex networks," IEEE Transactions on Computational Social Systems, vol. 6, no. 3, pp. 467-478, 2019.

[12] W. Li, L. Tian, and H. Batool, "Impact of negative information diffusion on green behavior adoption," Resources, Conservation and Recycling, vol. 136, pp. 337-344, 2018.

[13] P. Van Schaik, J. Jansen, J. Onibokun, J. Camp, and P. Kusev, "Security and privacy in online social networking: risk perceptions and precautionary behaviour," Computers in Human Behavior, vol. 78, pp. 283-297, 2018.

[14] C. Zhang and H. Huang, "Optimal control strategy for a novel computer virus propagation model on scale-free networks," Physica A: Statistical Mechanics and Its Applications, vol. 451, no. 451, pp. 251-265, 2016.

[15] C. Gan, Q. Feng, Q. Zhu, Z. Zhang, Y. Zhang, and Y. Xiang, "Analysis of computer virus propagation behaviors over complex networks: a case study of Oregon routing network," Nonlinear Dynamics, vol. 100, no. 2, pp. 1725-1740, 2020.

[16] J. Ren, Y. Xu, C. Xie, and S. He, "A compartmental model for global spread dynamics of malware under mutation," Mathematical Methods in the Applied Sciences, vol. 42, no. 6, pp. 1859-1869, 2019.

[17] C. Gan, Q. Feng, X. Zhang, Z. Zhang, and Q. Zhu, "Dynamical propagation model of malware for cloud computing security," IEEE Access, vol. 8, pp. 20325-20333, 2020.
[18] L. Yang, Z. Li, and A. Giua, "Containment of rumor spread in complex social networks," Information Sciences, vol. 506, pp. 113-130, 2020.

[19] L. Zhu, M. Liu, and Y. Li, "The dynamics analysis of a rumor propagation model in online social networks," Physica A: Statistical Mechanics and Its Applications, vol. 520, pp. 118137, 2019 .

[20] W. Wang, Q.-H. Liu, S.-M. Cai, M. Tang, L. A. Braunstein, and H. E. Stanley, "Suppressing disease spreading by using information diffusion on multiplex networks," Science Reports, vol. 6, 2016.

[21] X. Wang, X. Wang, F. Hao, G. Min, and L. Wang, "Efficient coupling diffusion of positive and negative information in online social networks," IEEE Transactions on Network and Service Management, vol. 16, no. 3, pp. 1226-1239, 2019.

[22] X. Wu, L. Fu, H. Long et al., "Adaptive diffusion of sensitive information in online social networks," IEEE Transactions on Knowledge and Data Engineering, vol. 1, 2020.

[23] L. Yang, Z. Li, and A. Giua, Rumor Containment by Spreading Correct Information in Social Networks, in Proceedings of the American Control Conference (ACC), pp. 5608-5613, New York, NY, USA, 2019.

[24] L.-X. Yang, P. Li, X. Yang, Y. Wu, and Y. Y. Tang, "On the competition of two conflicting messages," Nonlinear Dynamics, vol. 91, no. 3, pp. 1853-1869, 2018.

[25] Y. Yi, Z. Zhang, and C. Gan, "The outbreak threshold of information diffusion over social-physical networks," Physica A: Statistical Mechanics and Its Applications, vol. 526, pp. 112, 2019.

[26] Y. Xiao, C. Song, and Y. Liu, "Social hotspot propagation dynamics model based on multidimensional attributes and evolutionary games," Communications in Nonlinear Science and Numerical Simulation, vol. 67, pp. 13-25, 2019.

[27] Y. Lin, X. Wang, F. Hao et al., "Dynamic control of fraud information spreading in mobile social networks," IEEE Transactions on Systems, Man, and Cybernetics: Systems, vol. 67, pp. 1-14, 2019.

[28] Q. Sun, Y. Li, H. Hu, and S. Cheng, "A model for competing information diffusion in social networks," IEEE Access, vol. 7, pp. 67916-67922, 2019.

[29] Y. Yi, Z. Zhang, and C. Gan, "The effect of social tie on information diffusion in complex networks," Physica A: Statistical Mechanics and Its Applications, vol. 509, pp. 783-794, 2018.

[30] H. R. Thieme, "Asymptotically autonomous differential equations in the plane," Rocky Mountain Journal of Mathematics, vol. 24, no. 1, pp. 351-380, 1993.

[31] R. C. Robinson, An Introduction to Dynamical Systems: Continuous and Discrete, Pearson Prentice Hall, Upper Saddle River, NJ, USA, 2004.

[32] Z. Zhang, C. Wang, C. Gan, S. Sun, and M. Wang, "Automatic modulation classification using convolutional neural network with features fusion of SPWVD and BJD," IEEE Transactions on Signal and Information Processing Over Networks, vol. 5, no. 3, pp. 469-478, 2019.

[33] C. Gan, L. Wang, Z. Zhang, and Z. Wang, "Sparse attention based separable dilated convolutional neural network for targeted sentiment analysis," Knowledge-Based Systems, vol. 188, pp. 1-10, 2020.

[34] C. Gan, A. Saini, Q. Zhu, Y. Xiang, and Z. Zhang, "Blockchain-based access control scheme with incentive mechanism for ehealth systems: patient as supervisor," Multimedia Tools and Applications, vol. 188, 2020. 